\title{
ESCORRENTÍA Y EROSIÓN TRAS EL ABANDONO DE TIERRAS DE CULTIVO EN MONTAÑA: RESULTADOS DE LA ESTACIÓN EXPERIMENTAL “VALLE DE AÍSA"
}

\author{
T. LASANTA ${ }^{1}$, E. NADAL-ROMERO ${ }^{1,2}$, P. SerranO-Muela ${ }^{1}$, S. M. Vicente-SerRanO ${ }^{1}$ \\ \& J. M. GARCíA-RUIZ ${ }^{1}$ \\ 1 Instituto Pirenaico de Ecología (CSIC). Campus de Aula Dei. Apdo.13034- Zaragoza. \\ 2 Dep. Earth and Environmental Sciences, Physical and Regional Geography Research Group \\ K.U.Leuven, Celestijnenlaan 200 E, 3001 Heverlee (Belgium). \\ E-mail:fm@ipe.csic.es
}

\begin{abstract}
The main landscape feature in the Pyrenean middle mountain is the presence of hillslopes occupies by old cultivated fields already abandoned, affected by different stages of plant colonization. By means of experimental plots, this paper studies runoff generation and sediment yield in two types of abandoned fields: (i) sloping fields previously cultivated with cereal and frequently fertilized; and (ii) fields corresponding to shifting agriculture, with scarce conservation measures. The results obtained were compared to a dense shrub cover plot. The abandoned plots yield much more water and sediment than the dense shrub cover, which shows a lower variability in the hydromorphological response. The progressive plant colonization of the two abandoned plots favoured the occurrence of a negative trend in runoff and erosion. This paper also demonstrated that the shifting agriculture fields yield more water and erosion than sloping fields, because of difficulties for plant succession.
\end{abstract}

Keywords: Soil erosion; land abandonment; shifting agriculture; experimental plots; Mediterranean mountains; Pyrenees; Spain.

RESUMEN.- El principal rasgo paisajístico de la montaña media pirenaica es la presencia de extensas laderas ocupadas por antiguos campos de cultivo ya abandonados, en distintas fases de colonización vegetal. A partir de parcelas experimentales, este trabajo estudia la generación de escorrentía y la producción de sedimento en dos tipos de campos abandonados: (i) campos en pendiente previamente cultivados con cereal y abonados frecuentemente, y (ii) campos correspondientes a agricultura nómada, con escasas medidas de conservación. Los resultados se compararon con una parcela de matorral denso. Se ha comprobado que las dos parcelas abando- 
nadas producen mucha más agua y sedimento que el matorral, que además muestra una menor variabilidad en su respuesta hidromorfológica. La progresiva colonización vegetal de las dos parcelas abandonadas favorece la ocurrencia de una tendencia regresiva en la escorrentía y la erosión. Este trabajo demuestra también que los campos de agricultura nómada producen más agua y erosión debido a las mayores dificultades para que avance la sucesión vegetal.

Palabras clave: Erosión del suelo; campos abandonados; "articas"; parcelas experimentales; montañas mediterráneas; Pirineos; España.

\section{Introducción}

El crecimiento demográfico de los siglos XVIII y XIX, acompañado por el inicio de la decadencia del sistema trashumante obligó en las montañas españolas a roturar y cultivar laderas de escasas aptitudes agrícolas, con el fin de garantizar la alimentación de la población local y sustituir las rentas hasta entonces generadas por el ovino trashumante y la manufactura textil (GÓMEZ URDÁÑEZ, 1986; LASANTA, 1989; MORENO-FERNÁNDEZ, 1994). Extensas laderas fueron ocupadas por cultivos cerealistas, utilizando frecuentemente áreas muy pendientes, alejadas de los núcleos de población, con suelos poco fértiles y muy pedregosos, a veces con barbechos muy largos enmarcados en una agricultura nómada propia de territorios muy pobres (VIOLANT, 1949; GARCÍA-RUIZ, 1976; CALVO PALACIOS, 1977).

El descenso demográfico registrado en la montaña española a lo largo del siglo XX, muy especialmente entre las décadas de los cincuenta y ochenta, junto con la incorporación de la montaña a un mercado muy dinámico y competitivo y más amplio espacialmente, han dado lugar al efecto contrario: en las últimas décadas la explotación de la montaña se basa en la intensificación agrícola de sus mejores espacios, los fondos de valle y áreas próximas a los núcleos de población, y en el abandono de las laderas (GARCÍA-RUIZ \& LASANTA, 1990). Lo cierto es que los campos abandonados constituyen un elemento esencial del paisaje, tanto por la gran extensión que ocupan como por sus implicaciones ambientales y socioeconómicas (GARCÍA-RUIZ, 1988; POYATOS et al., 2003; ROMEROCALCERRADA \& PERRY, 2003; VICENTE-SERRANO et al., 2000; ERREA et al., 2007; LASANTA \& VICENTE-SERRANO, 2007; VILÀ-SUBIRÓS et al., 2009).

Los campos abandonados en pendiente, dominantes en el Pirineo occidental (LASANTA, 1996), muestran una gran variedad de ambientes 
geomorfológicos. Parcelas extremadamente erosionadas, con incisiones, flujos de derrubios y erosión difusa severa, alternan con otras en las que el matorral o una cubierta densa de herbáceas protegen el suelo, sin que se observen formas erosivas (RUIZ-FLAÑO, 1993). Esta diversidad de ambientes se ha justificado atendiendo, sobre todo, a la gestión que cada parcela tiene tras el abandono. Los resultados obtenidos por RUIZ-FLAÑO (1993) GARCÍA-RUIZ et al. (1991) y RUIZ FLAÑO et al. (1992) sugieren que los campos abandonados siguen dos tendencias evolutivas: Si la sucesión vegetal no se ve interrumpida por incendios o sobrepastoreo, el matorral llega a cubrir totalmente el suelo y la erosión se reduce al mínimo. Pero si la gestión humana dificulta la colonización vegetal, se instala un matorral abierto que permite la génesis de procesos de erosión difusa fuerte y descalzamientos, que en un estadio muy avanzado de degradación conducen a campos cubiertos completamente por pavimentos de piedras.

No cabe duda de que la gestión tras el cese del cultivo cumple un papel esencial en la evolución geomorfológica de los campos abandonados. No obstante, cabe plantearse ¿en qué medida la fase de cultivo condiciona la evolución posterior? La localización de los campos en laderas más o menos pendientes y con suelos de diferente profundidad y fertilidad, la aplicación o no de fertilizantes, los sistemas de laboreo utilizados, con barbechos más o menos largos, deben ser factores con implicaciones en la disponibilidad de nutrientes, en el proceso de sucesión vegetal y en la evolución de la escorrentía y la erosión del suelo. En la agricultura tradicional de montaña se distinguían cuatro modelos de campos (LASANTA, 1989): (i) llanos, ocupando los fondos de valle o rellanos colgados (antiguos depósitos de obturación glaciolacustre); (ii) campos en pendiente, adaptados a la forma e inclinación de la ladera, a veces con un ligero abancalamiento en la parte inferior; (iii) bancales o terrazas delimitadas por muros de piedra o saltos cubiertos de hierba y arbolado; y (iv) campos correspondientes a agricultura itinerante o nómada (articas, en la denominación local), cultivados durante dos o tres años seguidos y con un barbecho largo de hasta 30 años, ocupando laderas muy pendientes y sin medidas de conservación.

En este trabajo se estudia la evolución de la escorrentía y el transporte de sedimento en dos parcelas abandonadas y cultivadas anteriormente con cereal (cebada de invierno); una de ellas representa a los campos en pendiente, sujetos a abonado durante el periodo de cultivo, y la otra representa a un campo típico de agricultura nómada. Para ello se analiza la información recopilada en la Estación Experimental "Valle de Aísa" desde 1992 a 2008. 


\section{2. Área de estudio}

La parte experimental del estudio se ha llevado a cabo en el Valle de Aísa, Pirineo Central español (Fig. 1). Dos grandes unidades morfoestructurales dominan en el valle: al norte, las Sierras Interiores, de litología calcárea, han originado grandes escarpes debido tanto al dispositivo estructural y la resistencia del roquedo como al glaciarismo pleistoceno. Inmediatamente al sur se localiza el sector del flysch Eoceno, que ha dado lugar a una sucesión de colinas de divisorias suaves y vertientes regularizadas, afectadas localmente por abarrancamientos (GARCÍA-RUIZ \& PUIGDEFÁBREGAS, 1982).

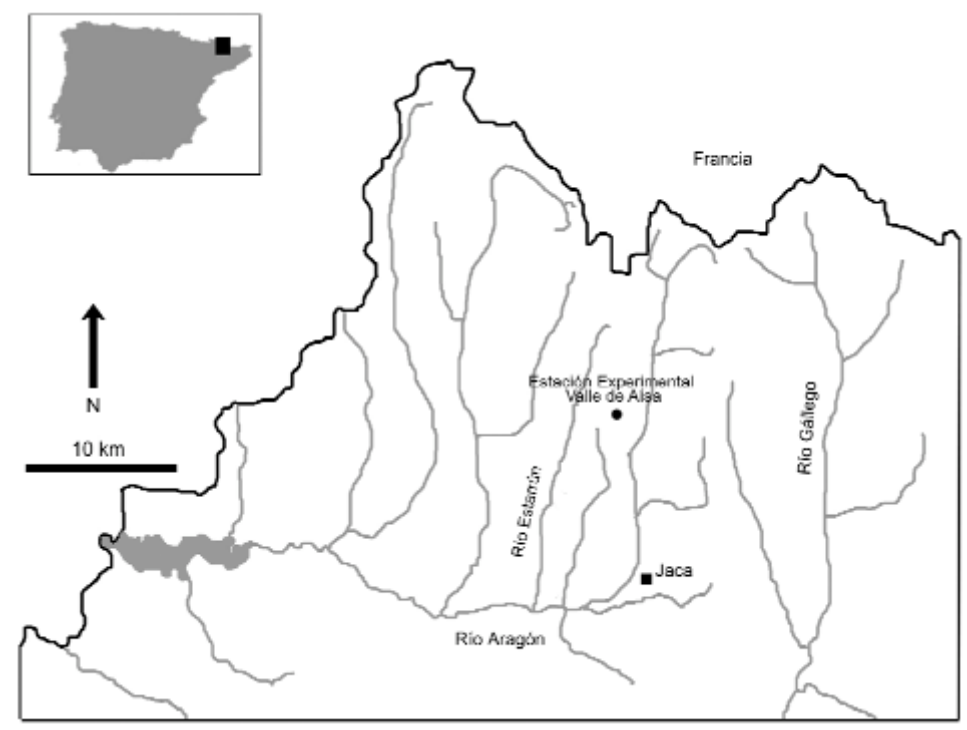

Figura 1. Localización de la Estación Experimental "Valle de Aísa". Figure 1. Location of the Aisa Valley Experimental Station.

En la localidad de Aísa, a 1100 m s.n.m., la precipitación media anual es de $1100 \mathrm{~mm}$, con una temperatura media anual de $10^{\circ} \mathrm{C}$. Por debajo de $1500 \mathrm{~m}$ s.n.m. los bosques de Pinus sylvestris dominan en las laderas umbrías, mientras que en las solanas coexisten pequeños rodales de Quercus gr. faginea con diversos matorrales submediterráneos (Genista scorpius, Rosa sp., Crataegus monogyna, Buxus sempervirens, Juniperus communis y Prunus spinosa constituyen las especies dominantes). El piso subalpino (por encima de los 
1700-1800 m s.n.m.) fue completamente deforestado desde la Edad Media para ampliar la superficie de pastos de verano (MONTSERRAT, 1992; GARCÍA-RUIZ \& VALERO, 1998). Los suelos son pardos, muy pedregosos y, en general, de escasa potencia, sobre todo en las solanas. Son pobres en nutrientes y en materia orgánica $(1,5 \%)$ y ricos en carbonato, por lo que su $\mathrm{pH}$ es siempre básico (RUIZ-FLAÑO, 1993).

El valle de Aísa ocupa una superficie de 8540 ha, de las que al menos 1749 ha (el 23,8\% de la extensión del Valle) se cultivaron hasta las primeras décadas del siglo XX. El espacio agrícola ocupaba el fondo del valle y la mayoría de las laderas solanas localizadas entre 900 y 1500 m s.n.m., con pendientes comprendidas entre el 20 y el $40 \%$. El cultivo principal era el cereal, que alternaba anualmente con el barbecho; una parte importante del espacio agrícola (643 ha) lo constituían las articas (campos típicos de una agricultura itinerante o nómada dedicados a cereal y fertilizados mediante la quema de matorrales), que se cultivaban durante 3-5 años para abandonarse a continuación durante 20-30 años; la práctica se reconocía como artigueo. En la actualidad se mantienen en cultivo 293 ha, localizándose los campos en los espacios llanos y de suave pendiente (parcelas que pueden ser trabajadas con maquinaria agrícola), donde los cereales han sido sustituidos por cultivos forrajeros (LASANTA, 1989). Entre 1930 y 1980 se abandonaron 1456 ha: la totalidad de las articas y los campos localizados en laderas más pendientes y alejados de los pueblos (LASANTA, 1988). Desde entonces todos los campos abandonados están inmersos en un proceso de sucesión vegetal: los campos en pendiente se encuentran mayoritariamente en la fase de cubrimiento denso de matorrales de Genista scorpius, principalmente, acompañada por Rosa gr. canina, Juniperus communis y Grataegus monogyna, mientras que en las articas abandonadas conviven pies aislados de matorral de Genista scorpius (aliaga) con herbáceas dispersas y áreas de enlosado de piedras (MOLINILLO et al., 1997; LASANTA et al., 2006).

\section{Métodos}

La Estación Experimental "Valle de Aísa" (EEVA) se localiza cerca del pueblo de Aísa (30TXN957285), a 1240 m s.n.m. en un antiguo campo de cultivo con el $32 \%$ de pendiente, abandonado a principios de los años sesenta y cubierto por matorral denso de Genista scorpius y Rosa gr. canina como especies más representativas.

En 1991 se instaló la Estación Experimental con nueve parcelas cerradas de $10 \times 3 \mathrm{~m}$, para controlar el comportamiento hidromorfológico de los usos y prácticas de la agricultura tradicional y actual del Pirineo: 
(i) Cereal fertilizado con abono químico.

(ii) Cereal de artigueo.

(iii) Barbecho.

(iv) Prado.

(v) Abandono después del cultivo con cereal fertilizado.

(vi) Abandono después del Cereal de artigueo.

(vii y viii) Dos parcelas quemadas sometidas a diferente frecuencia de incendios.

(ix) Matorral denso (vegetación natural inalterada).

Cada parcela dispone de un canal Gerlach en la parte baja, conectado a un sistema de balancines y a data loggers para registrar los eventos de escorrentía de forma continua. Además la estación cuenta con un pluviógrafo conectado a uno de los data loggers para registrar en continuo el volumen e intensidad de la lluvia. Parte de la escorrentía de cada parcela es desviada desde los balancines a depósitos de entre 130 y 220 litros de capacidad. Después de cada evento lluvioso se recoge una muestra de agua para conocer la exportación de sedimento en suspensión y los materiales disueltos (GARCÍARUIZ et al., 1995).

Anualmente se realizó un muestreo de vegetación siguiendo el método de la unidad de superficie. Un cuadrado de $50 \mathrm{~cm}$ de lado se lanzó tres veces dentro de cada parcela. A continuación se estimó el porcentaje de cubrimiento de herbáceas y matorrales, y se identificaron las especies más abundantes.

Para los objetivos de este trabajo se utiliza la información de tres parcelas:

i) Parcela abandonada en 1993, después de ser cultivada con cebada (PA1)

ii) Parcela abandonada en 1996, tras 4 años de cultivo con cebada bajo el sistema de artigueo (PA2). Para ello se procedió a desbrozar el matorral de Genista scorpius, quemarlo lentamente bajo un montón de tierra y distribuir las cenizas a modo de abono.

(iii) Parcela de matorral denso, con el fin de comparar las parcelas recientemente abandonadas con los ambientes ya estabilizados después de décadas de abandono.

Debe tenerse en cuenta el distinto grado de fertilización recibido por las parcelas abandonadas durante la fase de cultivo. En PA1 se distribuyó la proporción correspondiente a $300 \mathrm{~kg} \mathrm{ha}^{-1}$ del abono complejo 15-15-15 (15\% de Nitrógeno amoniacal, 15\% Anhídrido fosfórico y 15\% de Óxido potásico, más pequeñas proporciones de magnesio, azufre, hierro y manganeso), mientras que en PA2 se esparcieron las cenizas resultantes de quemar aliagas siguiendo el método descrito anteriormente. Este último sistema de fertilización aporta muy pocos nutrientes, ya que las cenizas sólo representan 
el 2,25\% de la planta, con un bajo contenido en $\mathrm{N}(1,24 \%), \mathrm{P}(0,05 \%), \mathrm{K}$ $(0,29 \%), \mathrm{Ca}(0,53 \%)$ y $\mathrm{Mg}(0,08 \%)$ (LASANTA et al., 2006).

Tres tipos de análisis estadísticos se han llevado a cabo: (i) se utilizaron los Diagramas de Caja para conocer el comportamiento medio y dispersión de los coeficientes de escorrentía y tasas de erosión en los tres usos del suelo analizados; (ii) para profundizar en las relaciones existentes entre los valores mensuales de precipitación y los coeficientes de escorrentía y las pérdidas de sedimento a escala mensual y anual se han aplicado correlaciones lineales de Pearson; y (iii) también se han realizado análisis estadísticos a partir de los residuales de la relación entre la precipitación y el coeficiente de escorrentía, por un lado, y las pérdidas de sedimento por otro (Sigmaplot 10 y SPPS 11).

\section{Resultados}

\subsection{Evolución del cubrimiento vegetal}

La figura 2 muestra la evolución de la cubierta vegetal en los tres usos del suelo analizados. PA2 dejó de cultivarse en 1996, tras cuatro años de cultivo bajo el sistema de artigueo. El proceso de sucesión vegetal pone de relieve que durante el primer año de abandono la proporción de suelo desnudo (68\% de la superficie del suelo) es muy superior al cubrimiento de herbáceas (32\%). Se trata de especies anuales muy oportunistas y de ciclo vital muy rápido (arvenses, principalmente, y ruderales en menor medida), que permanecieron en el banco de semillas del suelo desde la fase de cultivo (MONTSERRAT, 1990). A partir de esa cubierta inicial las herbáceas progresan de forma sostenida, llegando a alcanzar en cinco años el $100 \%$ del cubrimiento; entre las más representativas se incluyen Brachypodium pinnatum, Convulvulus arvensis, Medicago lupulina y Sanguisorba minor. Los matorrales (inicialmente algunas plántulas de Genista scorpius) penetran de forma muy tímida; en 2003 (8 años después del abandono) su presencia representaba sólo el 5\%: algunos pies aislados de aliaga en los márgenes de la parcela, que avanzaban de forma muy lenta, ya que en 2008 sólo cubrían el $15 \%$ del suelo.

PA1 presenta un avance más rápido de herbáceas y matorrales que la Artica Abandonada. El primer año de abandono las herbáceas cubren ya el $65 \%$ del suelo, alcanzando cinco años después el 100\%. Entre las especies más abundantes se incluyen: Brachypodium pinnatum, Dactylis glomerata, Centaurea jacea, Galium lucidum, Sanguisorba minor, Medicago sativa,... Los matorrales 
(Genista scorpius y Rosa sp.) cubrían el cuarto año de abandono el 8\%, el quinto el 15\% y en 2008 (16 años después del cese del cultivo) el 60\%, dando lugar a una cubierta vegetal bastante compleja con superposición de dos estratos: matorral y herbáceas.
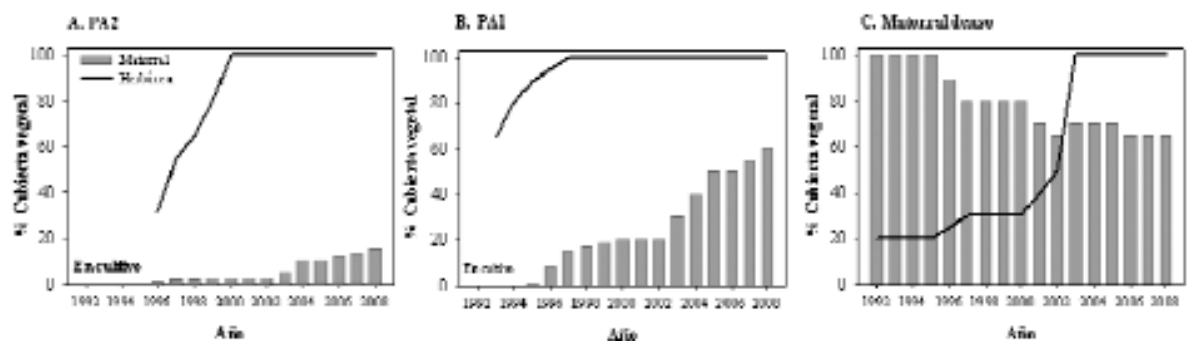

Figura 2. Evolución de la cubierta vegetal (1992-2008).

Figure 2. Evolution of the vegetation cover (1992-2008).

La parcela de Matorral denso presenta un buen cubrimiento durante los 17 años de estudio. No obstante, muestra algunos cambios interesantes. En 1992 los matorrales (Genista scorpius, Rosa sp., Juniperus communis y Grataegus monogyna) ocupaban el 100\%; la densidad era tan elevada que "ahogaban" a las herbáceas, que sólo estaban presentes en el 20\% de la superficie del suelo. Desde 1996 algunos pies de Genista scorpius mueren, disminuyendo lentamente su presencia, por lo que ya en 2008 los matorrales sólo cubrían el $65 \%$ del suelo. La formación de claros en el estrato arbustivo permite la progresión de las herbáceas, que pasan de ocupar el 20\% en 1992 al 30\% en $1997,50 \%$ en 2002 y $100 \%$ en 2008. Estos cambios implican que la cubierta vegetal de la parcela de Matorral denso sea muy parecida en la actualidad a la de la parcela Abandonada a partir de cereal fertilizado.

\subsection{Respuesta hidrológica y erosiva de las parcelas abandonadas durante el periodo} 1992-2008

La figura 3 refleja, mediante un gráfico de cajas, los coeficientes de escorrentía medios anuales y la pérdida media anual de sedimento. En el gráfico de la izquierda se observa que las parcelas abandonadas presentan coeficientes de escorrentía muy superiores a la parcela de Matorral denso. De hecho, la mediana de PA2 se sitúa próxima al valor 8 , mientras que en PA1 
está en 7 y en la de Matorral en 4. De igual forma, las cajas (percentiles entre 25 y 75) y los valores extremos están muy por encima en las parcelas abandonadas que en la parcela de Matorral denso. Esta última, por otra parte, muestra un rango de variación relativamente moderado, de manera que su valor más extremo está muy poco por encima de la mediana de PA2, reflejando que la variabilidad en las precipitaciones queda muy amortiguada por la presencia de una cubierta densa de matorral. Lo contrario ocurre en PA2, donde pueden registrarse valores muy extremos.

El gráfico correspondiente a la pérdida de sedimento refleja un comportamiento parecido al del coeficiente de escorrentía, con valores de la mediana más altos en la PA2 $\left(0.35 \mathrm{Mg} \mathrm{ha}^{-1} \mathrm{año}^{-1}\right)$, seguida de PA1 $(0.3 \mathrm{Mg}$ ha$\left.{ }^{1} \mathrm{año}^{-1}\right)$ y más modestos en la de Matorral denso $\left(0.08 \mathrm{Mg} \mathrm{ha}^{-1} \mathrm{año}^{-1}\right)$. Una cuestión interesante es que los valores de las medianas de las dos parcelas abandonadas derivan de distribuciones muy diferentes: así, PA1 presenta una caja muy alargada, mostrando una fuerte diversidad en las tasas anuales de erosión (percentiles del 25\% y 75\% con valores comprendidos entre $0.2 \mathrm{Mg}$ ha${ }^{1}$ año ${ }^{-1}$ y $1.7 \mathrm{Mg} \mathrm{ha}^{-1}$ año $\left.{ }^{-1}\right)$, mientras que PA2 dibuja una caja pequeña, por la baja variabilidad interanual (valores comprendidos entre $250 \mathrm{~kg} \mathrm{ha}^{-1}$ y $700 \mathrm{~kg}$ $\mathrm{ha}^{-1}$ ), si bien las tasas de erosión registradas algunos años son muy elevadas, situándose el percentil 90 en $4 \mathrm{Mg} \mathrm{ha}^{-1}$ año-1 $^{-1}$ y escapando la tasa más alta (5.1 $\mathrm{Mg} \mathrm{ha}^{-1} \mathrm{año}^{-1}$ ) de la escala gráfica de la figura. El gráfico correspondiente a la parcela de Matorral denso muestra que el C50 es más bajo que el C10 de las parcelas abandonadas y que el C90 apenas supera sus medianas. Además, dibuja una caja muy pequeña y unos valores externos próximos a la caja, poniendo de manifiesto un comportamiento muy estable a lo largo del tiempo.

La figura 4 muestra las regresiones entre la precipitación y la escorrentía a escala mensual. Las parcelas PA1 y Matorral denso presentan coeficientes de determinación similares $\left(R^{2}=0,32\right)$, mientras que la relación es más baja en PA2 $\left(R^{2}=0,21\right)$, aunque en los tres casos las relaciones son estadísticamente significativas al nivel 0,01. Las dos parcelas abandonadas muestran una nube de puntos muy dispersa alrededor de la recta de regresión, reflejando la notable variabilidad de la respuesta hidrológica. En cambio, en la parcela de Matorral denso los puntos aparecen mucho más ajustados en torno a la recta. De hecho, una misma precipitación tiene una respuesta muy diferente en las tres parcelas. Así, por ejemplo, una precipitación de $332 \mathrm{~mm}$ en enero de 2001 generó coeficientes de escorrentías muy contrastados: el valor más elevado se registró en PA2 (25\%), seguida de cerca por PA1 $(21 \%)$, y a mucha distancia por la de Matorral denso (12\%). 

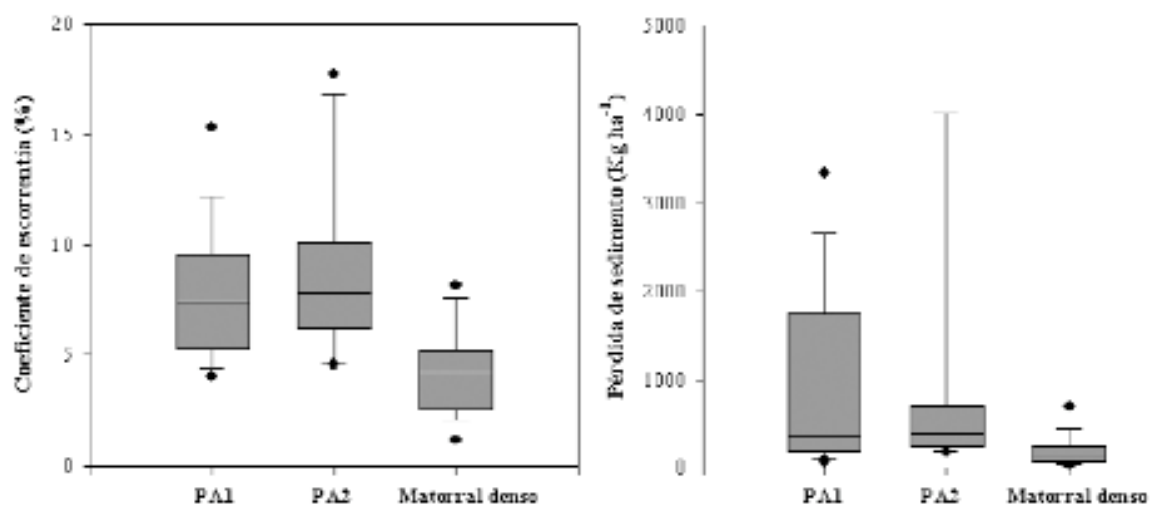

Figura 3. Diagrama de cajas sobre variabilidad del coeficiente de escorrentía y la pérdida de sedimento (1992-2008). Las barras indican los centiles 10 y 90. Los extremos de la caja los centiles 25 y 75. La línea interior de la caja es la mediana.

Figure 3. Box Plot about the varaibility of the runoff coefficient and the sediment yield (1992-2008). Bars show the $10^{\text {th }}$ and $90^{\text {th }}$ percentiles. The ends of the box indicate the $25^{\text {th }}$ and $75^{\text {th }}$ percentiles. The inner line in the box plot correspond with the median.
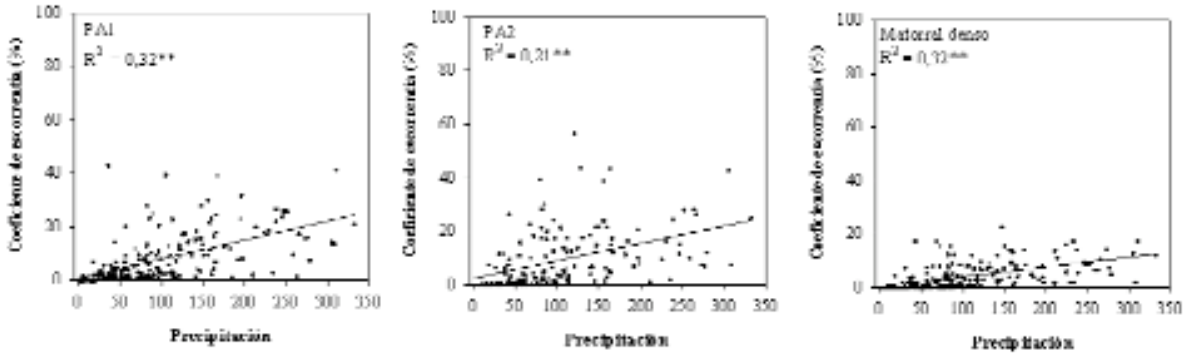

Figura 4. Coeficiente de correlación entre precipitación y coeficiente de escorrentía a escala mensual.

Figure 4. Monthly relationships between precipitation and runoff coefficient.

En la Fig. 5 se presentan las correlaciones entre la precipitación y la pérdida de sedimento mensual. En los tres casos las correlaciones son significativas al nivel 0,01. La correlación más elevada se registra en la parcela de Matorral denso $\left(R^{2}=0,35\right)$, muy por encima de la parcela PA1 $\left(R^{2}=0,19\right)$ y PA2 $\left(R^{2}=0,13\right)$.

Con el fin de observar la posible existencia de una tendencia temporal en las tres parcelas, especialmente en las abandonadas, se procedió primero a calcular los residuales procedentes de la relación entre la precipitación y el 

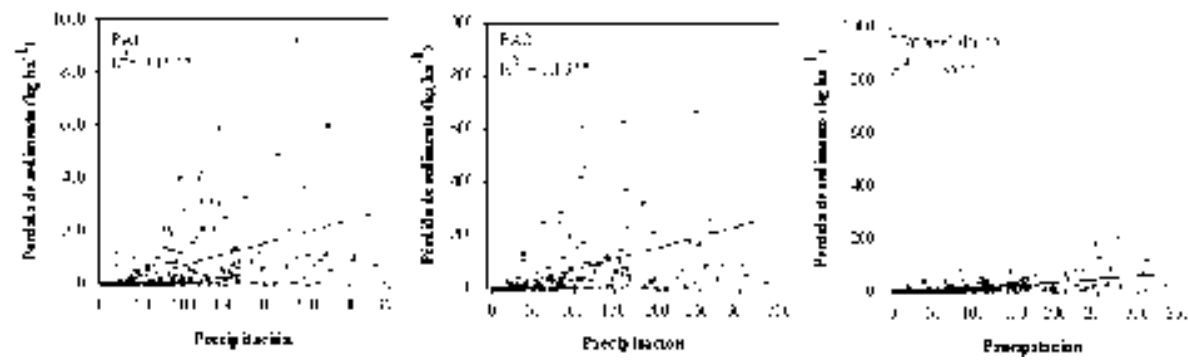

Figura 5. Coeficiente de correlación entre precipitación y pérdida de sedimento a escala mensual. Figure 5. Monthly relationships between precipitation and sediment yield.

coeficiente de escorrentía, para después relacionarlos con los años. Posteriormente se siguió el mismo procedimiento con las relaciones entre la precipitación y la pérdida de sedimento. En ambos casos, los análisis se llevaron a cabo a escala mensual y anual.

A escala mensual, los residuales de la relación entre precipitación y coeficiente de escorrentía no presentan la más mínima tendencia temporal, con unos coeficientes de determinación próximos a cero (Fig. 6). En cambio, sí muestran una notable tendencia regresiva los residuales de la relación entre precipitación y pérdida de sedimento (Fig. 7), especialmente en el caso de la Parcela de Cereal abandonado y PA2 ( $\mathrm{R}^{2}=0,6$ y 0,78 respectivamente), con un ligero repunte reciente en esta última. La parcela de Matorral denso muestra también una relación significativa, aunque con una tendencia menos apreciable en el gráfico debido a que los valores de los residuales son mucho más bajos.
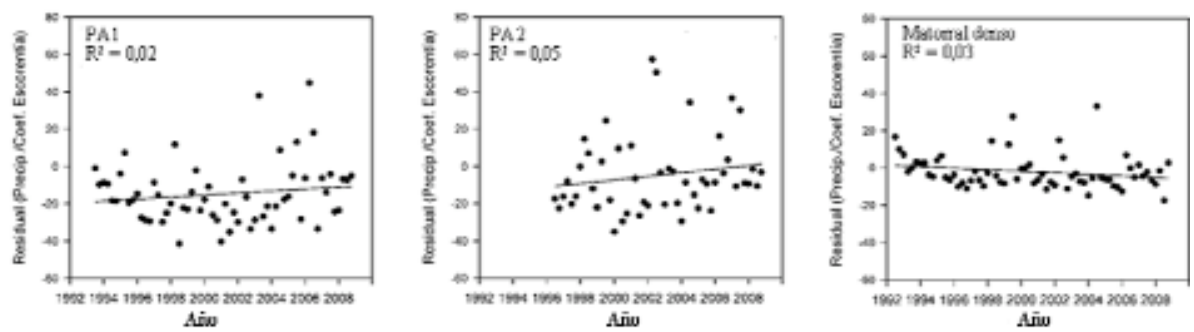

Figura 6. Tendencia en los residuales de la relación entre precipitación y el coeficiente de escorrentía a escala mensual

Figure 6. Monthly tendency in the residuals in the relationships between precipitation and runoff coefficient. 

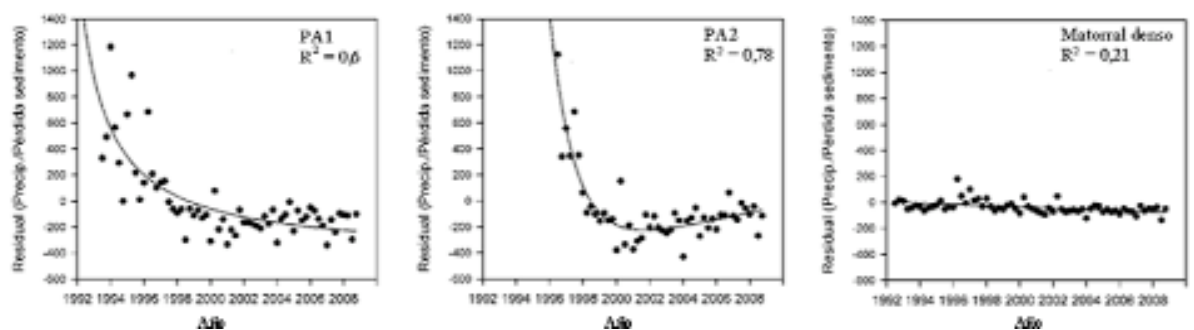

Figura 7. Tendencia en los residuales de la relación entre precipitación y erosión del suelo a escala mensual.

Figure 7. Monthly tendency in the residuals in the relationships between precipitation and sediment yield.

A escala anual se registra tendencia temporal para los residuales entre precipitación y coeficiente de escorrentía (Fig. 8), de manera que este último es progresivamente menor a medida que pasa el tiempo en las dos parcelas abandonadas. La parcela de Matorral denso no presenta, en cambio, tendencia. Al contrario de lo que sucedía a escala mensual, los residuales entre precipitación y producción de sedimento no muestran ninguna significación en su relación con el tiempo (Fig. 9).

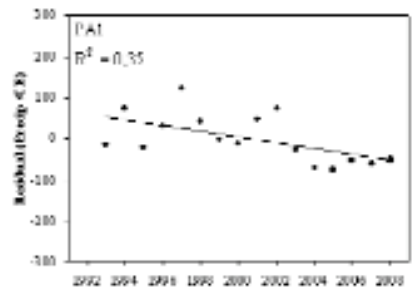

Als

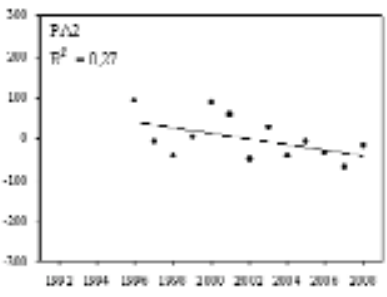

Asto

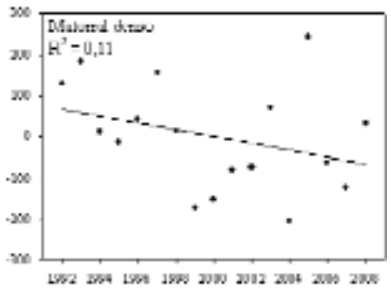

Ath

Figura 8. Tendencia en los residuales de la relación entre precipitación y el coeficiente de escorrentía a escala anual.

Figure 8. Annual tendency in the residuals in the relationships between precipitation and runoff coefficient. 


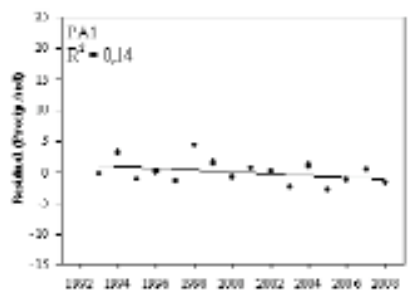

Aste

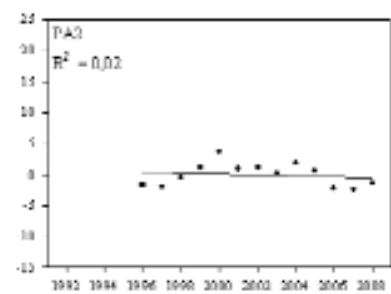

An

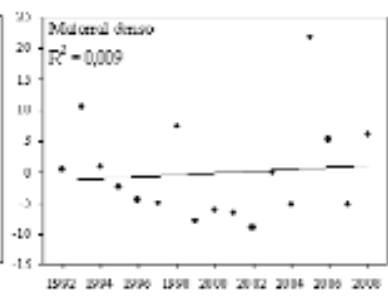

Anto

Figura 9. Tendencia en los residuales de la relación entre precipitación y erosión del suelo a escala anual.

Figure 9. Annual tendency in the residuals in the relationships between precipitation and sediment yield.

\section{Discusión y conclusiones}

Las áreas de montaña han experimentado importantes cambios en la superficie cultivada, cuya extensión se halla muy relacionada con la densidad de población (LASANTA, 1990; RUIZ-FLAÑO, 1993; MCDONALD et al., 2000; POYATOS et al., 2003). En el caso del Pirineo y de otras montañas españolas la máxima expansión del espacio cultivado se alcanzó a lo largo del siglo XIX. La rápida despoblación que tuvo lugar desde principios de siglo $\mathrm{XX}$ y, especialmente, desde los años cincuenta, provocó un rápido proceso de abandono que afectó primero a los peores campos y que actualmente limita la superficie cultivada a menos del 3\% de la total. Tras el abandono, los antiguos campos cultivados entraron en una etapa compleja de colonización vegetal relacionada con la calidad del suelo y el tipo de gestión posterior al abandono (pastoreo extensivo, incendios periódicos) (RUIZ-FLAÑO, 1993; MOLINILLO et al., 1997). Desde una perspectiva geoecológica, el problema crítico consiste en relacionar la colonización vegetal (diferente según el modelo de campo), la generación de escorrentía y la producción de sedimento, y analizar la evolución temporal de esas relaciones. No debe olvidarse que las áreas de montaña son las principales generadoras de escorrentía en el mundo mediterráneo (VIVIRIOLI et al., 2003) y que la vegetación puede ser un factor tan importante o más que el clima a la hora de explicar la evolución de los recursos hídricos (BEGUERÍA et al., 2003; ANDRÉASSIAN, 2004). Por otra parte, el ritmo de aterramiento de los embalses en montaña está condicionado por la presencia o no de una cubierta vegetal densa y por la localización de las fuentes de sedimento en relación con los cauces fluviales (conectividad o accesibilidad) (BEGUERÍA, 2005). Este trabajo se ha centrado en la evolución hidromorfológica de dos modelos de campos después de haberse abandonado: campos en pendiente (PA1), que 
eran los más frecuentes en el Pirineo Occidental, y campos de agricultura nómada (articas) (PA2), que se cultivaban en ambientes más marginales desde el punto de vista de la pendiente y la calidad del suelo, sin apenas medidas de conservación. Para ello se ha dispuesto de la información procedente de la Estación Experimental "Valle de Aísa", utilizando una tercera parcela, cubierta de matorral denso, como referente comparativo.

Los resultados obtenidos demuestran que la producción de agua y sedimento es muy superior en las dos parcelas abandonadas que en la parcela de Matorral denso, sobre todo al inicio del experimento, debido al tiempo necesario para que se establezca una cubierta de herbáceas y comience la colonización por parte de matorrales. Esto es especialmente cierto en el caso de PA2, en la que la penetración de la vegetación ha sido más lenta debido a las condiciones más extremas de su cultivo y al bajo contenido en nutrientes del suelo. Además, ambas parcelas muestran una respuesta hidromorfológica extremadamente variable, de manera que dicha respuesta depende aún mucho de la intensidad y volumen de las precipitaciones y del estado de humedad del suelo. Por el contrario, la parcela de Matorral denso está mucho más condicionada en su respuesta por la presencia de una cubierta estable de matorral con un sustrato de herbáceas, lo que amortigua mucho el efecto de la variabilidad de la precipitación. No cabe duda, en todo caso, de que PA1 produce menos agua y sedimento que PA2, reflejando que el comportamiento hidromorfológico tras el abandono no es independiente de los modelos de campos o del tipo de gestión mientras estaban en cultivo (GARCÍA-RUIZ \& LÓPEZ BERMÚDEZ, 2009).

La evolución temporal de las dos parcelas abandonadas presenta resultados un tanto sorprendentes. De entrada, lo más importante es que existe una tendencia temporal regresiva, que se manifiesta de manera diferente a escala mensual y anual. El empleo de los residuales de la relación entre precipitación y escorrentía, por un lado, y precipitación y producción de sedimento, por otro, demuestra que a escala mensual sólo las pérdidas de sedimento muestran una clara tendencia temporal y no, como también cabría esperar, la generación de escorrentía. Por el contrario, a escala anual es la escorrentía la que presenta tendencia temporal, y no la producción de sedimento. Esto confirma que las relaciones entre precipitación y generación de escorrentía se establecen mejor a escalas temporales largas, mientras que en las cortas hay demasiada influencia del estado de humedad del suelo antecedente y de las precipitaciones que hayan podido ocurrir en semanas o meses anteriores. No sucede lo mismo con el sedimento, que está más relacionado con las lluvias de cada mes (intensidad y volumen) y no con el volumen anual de precipitaciones, que refleja muy poco los rasgos individuales de los eventos pluviométricos. No debe olvidarse, por ejemplo, 
que una proporción muy importante del sedimento transportado cada año depende de dos o tres eventos lluviosos (GONZÁLEZ-HIDALGO et al., 2007) y no tanto de la cantidad total de lluvia anual.

En todo caso, es innegable que las parcelas abandonadas muestran una tendencia temporal, con una progresiva reducción de la producción de escorrentía y sedimento. Esta tendencia refleja la colonización vegetal que afecta a las parcelas desde el momento en que los campos dejan de cultivarse, favoreciendo un creciente consumo de agua por la vegetación, una mayor infiltración y una reducción de los procesos de erosión. En cambio, la parcela de Matorral denso no presenta tendencia temporal alguna, dado que los cambios de vegetación que hayan podido darse en esa parcela son, por el momento, de escasa relevancia desde un punto de vista hidromorfológico. De todas formas, la ocurrencia de un claro proceso de senescencia en Genista scorpius podría dar lugar a corto plazo a un aumento de la escorrentía y la erosión. Sí es interesante comprobar que los residuales de la relación entre precipitación y escorrentía a escala mensual experimentan en PA2 un cambio de signo en los últimos años, con un ligero incremento a partir de 2000, después de haberse caracterizado por una brusca disminución entre 1996 y 2000. El significado de esta evolución no está, por el momento, claro, aunque probablemente se debe a las dificultades que encuentra la vegetación para instalarse sobre un suelo deteriorado y sujeto a fuerte erosión como el de la artica mientras estuvo en cultivo.

La reinstalación de la cubierta vegetal en campos abandonados es una dinámica muy compleja en función de las condiciones ambientales, la fertilidad de cada campo y la gestión previa y posterior al abandono (RUIZFLAÑO, 1993; PADILLA, 1998; GALLEGO-FERNÁNDEZ et al., 2004). En este trabajo se ha comprobado que, si bien a grandes rasgos el proceso de colonización tiene muchos puntos en común entre las dos parcelas abandonadas, el ritmo temporal es muy diferente con avances más rápidos en PA1 que en PA2; así, durante el primer año de abandono la PA2 sólo llega a alcanzar un cubrimiento del $32 \%$ por un $65 \%$ en PA1. Por otro lado, trece años después del abandono los matorrales sólo cubren el 15\% de la superficie en PA2 por un $50 \%$ en PA1.

Las diferencias en la evolución del cubrimiento se explican por el manejo del suelo, sobre todo la fertilización, en la fase de cultivo. La mayor riqueza de nutrientes en la parcela PA1 hace que pronto se instalen algunas arvenses anuales, ya presentes en el banco de semillas, cubriendo buena parte de la parcela. A partir de ahí, progresan rápidamente conviviendo especies anuales con otras perennes; de forma simultánea, las aliagas penetran desde los márgenes de la parcela hacia el interior avanzando sin grandes dificultades dada la elevada disponibilidad de nutrientes en el suelo. En la artica, por el 
contrario, la progresión de las aliagas se ve muy limitada por el bajo contenido de nutrientes en el suelo, sobre todo de nitrógeno, lo que reduce la producción de semillas (DE STEVEN, 1982; MONTSERRAT, 1990).

\section{Agradecimientos}

Este trabajo ha contado con el apoyo de los proyectos de investigación PROBASE (CGL2006-11619/HID), CETSUS (CGL2007-66644-C04-03/HID) y FIREGLOBE (CGL2008-01083/CLI), financiados por la Comisión Interministerial de Ciencia y Tecnología, ACQWA (FP7-ENV-2007-1), financiado por la Comisión Europea, y PI032/08, financiado por el Gobierno de Aragón. Ha contado también con la ayuda del Ministerio de Medio Ambiente, Medio Rural y Marino, a través de la RESEL y del Gobierno de Aragón mediante el "Programa de Grupos de Investigación Consolidados". La contribución de E. Nadal-Romero ha sido posible gracias a la financiación del Ministerio de Educación y Ciencia, mediante el Programa Nacional de Movilidad de Recursos Humanos del Plan Nacional de I-D+I 2008-2011. La contribución de P. Serrano-Muela ha sido posible gracias a la beca predoctoral I3P concedida por el Consejo Superior de Investigaciones Científicas (CSIC).

\section{Referencias}

ANDRÉASSIAN, V., 2004. Waters and forests: from historical controversy to scientific debate. Journal of Hydrology, 291 (1-2): 1-27. doi:10.1016/j.jhydrol.2003.12.015.

BEGUERÍA, S., 2005. Erosión y fuentes de sedimento en la cuenca del embalse de Yesa (Pirineo Occidental): Ensayo de una metodología basada en teledetección y análisis SIG. Instituto Pirenaico de Ecología (CSIC), 158 pp., Zaragoza.

BEGUERÍA, S., LÓPEZ-MORENO, J.I., LORENTE, A., SEEGER, M. \& GARCÍA-RUIZ, J.M., 2003. Assessing the effect of climate oscillations and land-uses in the Central Spanish Pyrenees. Ambio, 32 (4): 283-286.

CALVO PALACIOS, J.L., 1977. Los Cameros. De región homogénea a espacio-plan. Instituto de Estudios Riojanos, 2 vols, Logroño.

DE STEVEN, D., 1982. Seed reproduction and seed mortality in a temperate forest shrub (witch-hazel, Hamamelis virginia). Journal Ecology, 70: 437-443.

ERREA, M.P., ARNÁEZ, J., ORTIGOSA, L., OSERÍN, M., RUIZ-FLAÑO, P. \& LASANTA, T., 2007. Marginación y paisaje en una montaña submediterránea (1956-2001): El ejemplo de Camero Viejo (Sistema Ibérico, La Rioja). Nimbus, 19-20: 53-70. 
ESCORRENTÍA Y EROSIÓN TRAS EL ABANDONO DE TIERRAS DE CULTIVO EN MONTAÑA:...

GALLEGO-FERNÁNDEZ, J.B., GARCÍA-MORA, M.R. \& GARCÍA-NOVO, F., 2004. Vegetation dynamics of Mediterranean shrublands in former cultural landscape at Grazalema mountains, South Spain. Plant Ecology, 172 (1): 83-94. doi: 10.1023/B:VEGE.0000026039.00969.7 .

GARCÍA-RUIZ, J.M., 1976. Modos de vida y niveles de renta en el Prepirineo del Alto Aragón Occidental. Monografías del Instituto de Estudios Pirenaicos, 106: 272 pp., Jaca (Huesca).

GARCÍA-RUIZ, J.M., 1988. La evolución de la agricultura de montaña y sus efectos sobre la dinámica del paisaje. Revista de Estudios Agro-Sociales, 146: 7-37.

GARCÍA-RUIZ, J.M. \& LASANTA, T., 1990. Land-use changes in the Spanish Pyrenees. Mountain Research and Development, 10 (3): 267-279.

GARCÍA-RUIZ, J.M. \& LÓPEZ-BERMÚDEZ, F., 2009. La erosión del suelo en España. Sociedad Española de Geomorfología 441 pp., Zaragoza.

GARCÍA-RUIZ \& PUIGDEFÁBREGAS, J., 1982. Formas de erosión en el flysch eoceno surpirenaico. Cuadernos de Investigación Geográfica, 8: 83-126.

GARCÍA-RUIZ, J.M., RUIZ-FLAÑO, P., LASANTA, T., MONTSERRAT, G., MARTÍNEZ-RICA, J.P. \& PARDINI, G., 1991. Erosion in abandoned fiels, what is the problem? En: Soil Erosion Studies in Spain (M. Sala, J.L. Rubio y J.M. García-Ruiz, Eds). Geoforma Ediciones: 97-108, Logroño.

GARCÍA-RUIZ, J.M., LASANTA, T., ORTIGOSA, L., RUIZ-FLAÑO, P., MARTÍ, C. \& GONZÁLEZ, C., 1995. Sediment yield under different land uses in the Spanish Pyrenees. Mountain Research and Development, 15 (3): 229-240.

GARCÍA-RUIZ, J.M. \& VALERO, B., 1998. Historical geomorphic processes and human activities in the Central Spanish Pyrenees. Mountain Research and Development, 18 (4): 309-320.

GÓMEZ-URDÁÑEZ, J.L., 1986. Subsistencia y descapitalización en el Camero Viejo al final del Antiguo Régimen. Brocar, 12: 103-140.

GONZÁLEZ-HIDALGO, J.C., PEÑA-MONNÉ, J.L. \& DE LUÍS, M., 2007. A review of daily soil erosion in Western Mediterranean areas. Catena, 71 (2): 193-199. doi:10.1016/j.catena.2007.03.005.

LASANTA, T., 1988. The process of desertion of cultivated areas in the Central Spanish Pyrenees. Pirineos, 132: 15-36.

LASANTA, T., 1989. Evolución reciente de la agricultura de montaña: el Pirineo Aragonés. Geoforma Ediciones: 220 pp., Logroño.

LASANTA, T. (1990). Tendances actuelles de l'organisation spatiale des montagnes espagnoles. Annales de Géographie, 551: 51-71.

LASANTA, T. (1996). La transformación del paisaje en montaña media por la actividad agrícola en relación con las condiciones ambientales. En: Acción humana y desertificación en ambientes mediterráneos (J.M. García-Ruiz y P. 
López-García, Eds). Consejo Superior de Investigaciones Científicas: 145172, Zaragoza.

LASANTA, T., BEGUERÍA, S. \& GARCÍA-RUIZ, J.M., 2006. Geomorphic and hydrological effects of traditional shifting agriculture in a Mediterranean Mountain Area, Central Spanish Pyrenees. Mountain Research and Development, 26 (2): 146-152.

LASANTA, T. \& VICENTE-SERRANO, S.M., 2007. Cambios en la cubierta vegetal en el Pirineo aragonés en los últimos 50 años. Pirineos, 162: 125154.

MACDONALD. D., CRABTREE, J.R., WIESINGER, G., DAX, T., STAMOU, N., FLEURY, P., GUTIÉRREZ-LAZPITA, J. \& GIBON, A., 2000. Agricultural abandonment in mountain areas of Europe: Environmental consequences and policy response. Journal of Environmental Management, 59 (1): 47-69. doi:10.1006/jema.1999.0335.

MOLINILLO, M., LASANTA, T. \& GARCÍA-RUIZ, J.M., 1997. Managing mountainous degraded landscapes after farmland abandonment in the Central Spanish Pyrenees. Environmental Management, 21 (4): 587-598.

MONTSERRAT, G., 1990. Estudio de la colonización vegetal de los campos abandonados del Valle de Aísa (Jaca, Huesca). Informe del Proyecto LUCDEME: Erosión y colonización vegetal en campos abandonados: 77 pp., Jaca.

MONTSERRAT, J., 1992. Evolución glaciar y postglaciar del clima y la vegetación en la vertiente sur del Pirineo: estudio palinológico. Monografías del Instituto Pirenaico de Ecología, 6: 149 pp., Jaca.

MORENO-FERNÁNDEZ, J.R., 1994. El monte público en La Rioja durante los siglos XVIII y XIX: aproximación a la desarticulación del régimen comunal. Gobierno de La Rioja: 295 pp., Logroño.

PADILLA, A., 1998. Colonización vegetal en campos abandonados de la provincia de Alicante. Publicaciones de la Universidad de Alicante: 386 pp., Alicante.

POYATOS, R., LATRON, J. \& LLORENS, P., 2003. Land-use and land cover change after agricultural abandonment. The case of a Mediterranean Mountain Area (Catalan Pre-Pyrenees). Mountain Research and Development, 23 (4): 52-58.

ROMERO-CALCERRADA, R. \& PERRY, G.L.W., 2003. The role of land abandonment in landscape dynamics in the SPA "Encinares del río Alberche y Cofio, Central Spain, 1984-1999". Landscape and Urban Planning, 66 (4): 217-232. doi:10.1016/S0169-2046(03)00112-9.

RUIZ-FLAÑO, P., 1993. Procesos de erosión en campos abandonados del Pirineo. Geoforma Ediciones: 191 pp., Logroño.

RUIZ-FLAÑO, P., GARCÍA-RUIZ, J.M. \& ORTIGOSA, L., 1992. Geomorphological evolution of abandoned fields. A case study in the 
ESCORRENTÍA Y EROSIÓN TRAS EL ABANDONO DE TIERRAS DE CULTIVO EN MONTAÑA:...

Central Pyrenees. Catena, 19 (3-4): 301-308._doi:10.1016/0341-8162 (92)90004-U.

VICENTE-SERRANO, S., LASANTA, T. \& CUADRAT, J.M., 2000. Transformaciones en el paisaje pirenaico como consecuencia del abandono de las actividades económicas tradicionales. Pirineos, 155. 111-133.

VILÀ-SUBIRÓS, J., RIBAS PALOM, A., VARGA LINDE, D. \& LLAUSÀS PASCUAL, A., 2009. Medio siglo de cambios paisajísticos en la montaña mediterránea: percepción y valoración social de la Alta Garrotxa (Girona). Pirineos, 164: 69-92.

VIOLANT, R., 1949. El Pirineo español. Editorial Plus Ultra: 675 pp., Madrid.

VIVIRIOLI, D., WEINGARTNER, R \& MESSERLI, B., 2003. Assessing the hydrological significance of the World's mountains. Mountain Research and Development, 23: 32-40. 